\title{
Rashes following cesarean delivery: a case report
}

\author{
Jiwei Huang", , Ximei Zhu", Caiyun Jiang, Zhuying Liu, Weifeng Zheng \\ Department of Pharmacy, The Third Affiliated Hospital of Sun Yat-sen University, Guangzhou, China \\ \#These authors contributed equally to this work as co-first authors. \\ Correspondence to: Jiwei Huang. Department of Pharmacy, The Third Affiliated Hospital of Sun Yat-sen University, Guangzhou, China. \\ Email: 947543512@qq.com.
}

\begin{abstract}
This work reports a case of rashes on multiple parts of the body following cesarean delivery caused by routine use of cefuroxime sodium and morphine during the perioperative period. A 29-yearold woman underwent a lower segment cesarean section under combined spinal-epidural anesthesia. During surgery, cefuroxime sodium was administered intravenously following the division of the umbilical cord for the prevention of infection. Morphine hydrochloride was given for analgesia at the end of the procedure. Rashes and pruritus appeared on the patient's abdomen, back and thighs. Cephalosporins and opioids may cause rashes and pruritus. According to the patient's physical signs and the time of drug injection, we considered the rash and pruritus adverse reactions caused by cefuroxime sodium and morphine hydrochloride. After the discontinuation of the medications, antiallergic treatment and the other treatment of symptoms, the patient's symptoms gradually subsided. To this end, we speculate that cefuroxime sodium or morphine hydrochloride cause adverse reactions, including rashes in patients. Physicians, nurses, and clinical pharmacists should closely observe patients who receive these medications. The medications should be at once stopped if an adverse reaction occurs.
\end{abstract}

Keywords: Cefuroxime sodium; morphine hydrochloride; rash; case report

Submitted Jul 16, 2020. Accepted for publication Sep 25, 2020.

doi: 10.21037/apm-20-1701

View this article at: http://dx.doi.org/10.21037/apm-20-1701

\section{Introduction}

Cesarean section is a procedure involving a class II surgical wound. Common pathogens are gram-negative bacilli, Enterococcus, Group B streptococci, and anaerobic bacteria. The "Guiding Principles for Clinical Application of Antimicrobial Drugs" (2015 Edition) recommends using preventive antimicrobials, including cefazolin sodium and cefuroxime sodium with or without metronidazole to prevent surgical site infection. Analgesia with morphine hydrochloride after cesarean section is a common management approach. Epidural morphine hydrochloride can supply long-term postoperative analgesia and is usually used for analgesia after cesarean section. In the present case, the patient developed rashes after routine use of morphine hydrochloride and cefuroxime sodium. From the patient's physical signs and the time of drug injection, we consider the rashes an adverse reaction to cefuroxime sodium and morphine hydrochloride. After the discontinuation of medications and the treatment of symptoms, these symptoms subsided. Earlier studies have reported that cefuroxime, sodium, and morphine hydrochloride all can cause adverse reactions, including rash or pruritus in different populations. This article reports a case of cesarean section maternal skin rash and pruritus using cefuroxime sodium and morphine hydrochloride. Clinical attention should be paid to adverse events in special populations. This article supplies references for clinical treatment of this type of adverse reaction.

We present the following case in accordance with the

^ORCID: 0000-0002-7647-5155. 

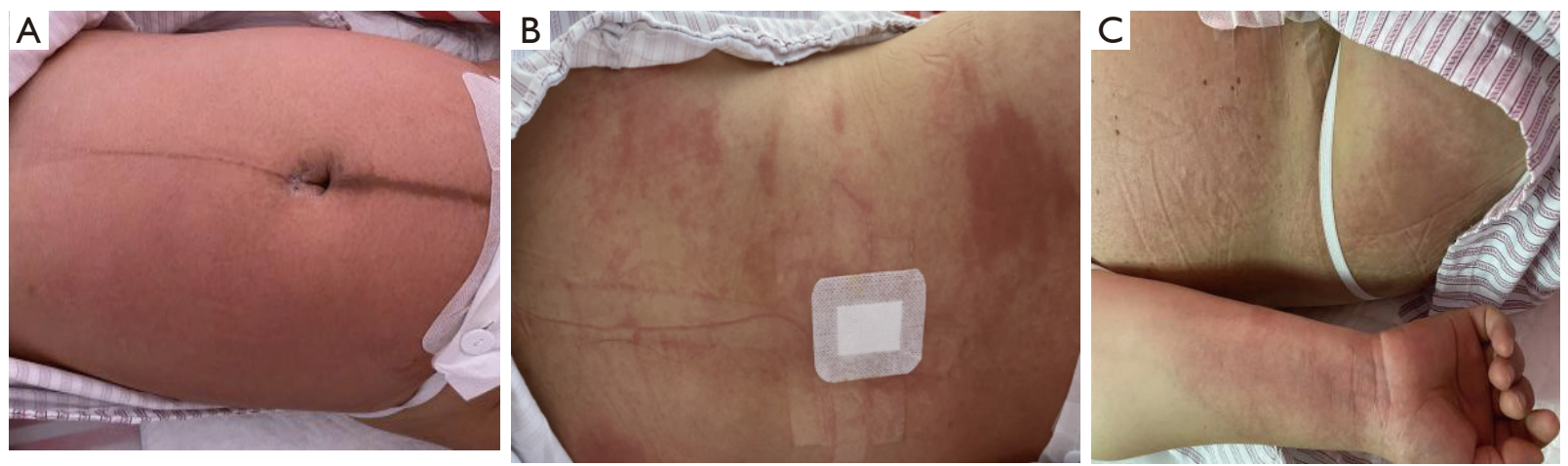

Figure 1 On the first day after surgery, the patient complained of rashes on (A) the abdomen, (B) the back, (C) the hands and legs.

CARE reporting checklist (available at http://dx.doi. org/10.21037/apm-20-1701).

\section{Case presentation}

\section{Patient data}

A 29-year-old woman was admitted to the Department of Obstetrics on May 16, 2020, due to "amenorrhea for 38 weeks, requesting surgery to terminate the pregnancy". She denied a history of chronic diseases, including hypertension, diabetes, and coronary heart disease, and a history of transmissible diseases, including hepatitis and tuberculosis. She had no history of food or drug allergy. She had never received a blood transfusion and did not receive scheduled immunization. Physical examination results were as follows: uterine height: $33 \mathrm{~cm}$; abdominal circumference: $99 \mathrm{~cm}$; fetal position: breech presentation; fetal heart rate: 140 beats/min. The fetal membranes were not broken. The results of biochemical and coagulation tests were within normal limits. On admission, the patient was at 38 weeks gestation. The fetus was in the breech presentation, and thus the patient underwent a lower segment cesarean section under combined spinalepidural anesthesia on May 17, 2020. During surgery, cefuroxime sodium (lot number 20011618, Shenzhen Lijian Pharmaceutical Co., Ltd.) was administered intravenously following the division of the umbilical cord for the prevention of infection. Morphine hydrochloride (lot number 190310-1, Northeast Pharmaceutical Group, Shenyang First Pharmaceutical Co., Ltd.) was given via the epidural space for analgesia at the end of the procedure. Then, morphine hydrochloride $(7 \mathrm{mg})$ plus ropivacaine hydrochloride injection (Naropin, $135 \mathrm{mg}$ ) was supported by an analgesic pump, intramuscular injection of carprost aminobutanol $250 \mathrm{~g}$ to stop bleeding. After surgery, the patient reported no significant discomfort and was sent back to the ward. The patient returned to the ward and complained of mild itching in the skin of the waist. At night, the pharmaceutical therapy regimen included intravenous administration of cefuroxime sodium $(0.75 \mathrm{~g}$ single dose), oxytocin injection (20 IU), vitamin C injection (2 g), coenzyme Q10 and sodium chloride injection $(250 \mathrm{~mL})$, and compound amino acid injection (18AA-VII, $200 \mathrm{~mL}$ ) for anti-inflammation, hemostasis, enhancement of uterine contractility and nutritional support.

On the first day after surgery, the patient complained about the pruritus on the waist, abdomen, back, and thighs, which was worse than before and unbearable (see Figure 1). No chills, fever, dizziness, headache, or cough with sputum were noted. She experienced occasional contraction pain. The urinary catheter was secured in place and unobstructed. The urine color was clear, and the urine output standard. She had no anal gas evacuation or bowel movement. Physical examination showed that her vital signs were stable. Rashes and scratch marks were visible on the abdomen, back, and thighs. Heart and lung examinations were unremarkable. She had no breast engorgement. The abdominal incision was healing well without redness, swelling, and exudation. The patient's uterine fundus was a finger-width above the umbilical naves. She had good uterine contractility and large volumes of bloody lochia. The urine examination was normal.

Routine blood examination showed a total leukocyte count of $9.87 \times 10^{9} / \mathrm{L}$, a hemoglobin concentration of $114.000 \mathrm{~g} / \mathrm{L}$, an average platelet volume of $9.700 \mathrm{fL}$, the neutrophil-to-lymphocyte ratio of 0.7990 , and a blood potassium level of $3.200 \mathrm{mmol} / \mathrm{L}$. Postoperative pruritus in the patient, considering that cefuroxime sodium and morphine hydrochloride are more likely to 

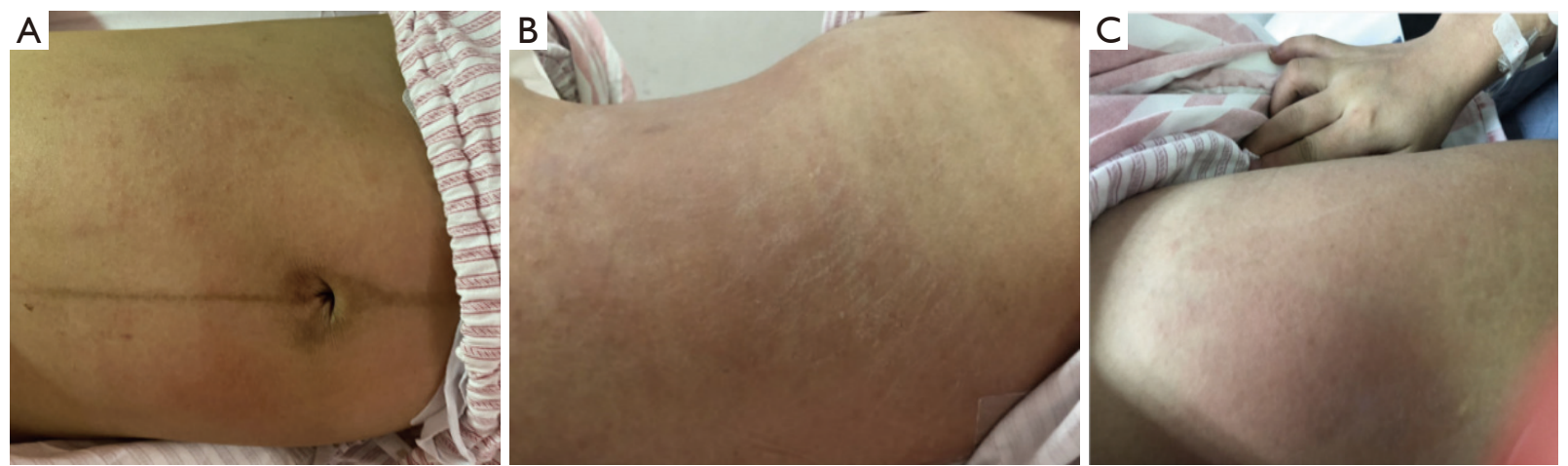

Figure 2 The rashes had almost disappeared on the fourth day after surgery. (A) Abdominal rashes had disappeared. (B) The rashes on the patient's back had disappeared. (C) The rashes on the hands and legs have disappeared.

cause drug allergy, using these two drugs, was suspended at present. And it was treated with loratadine tablets (10 $\mathrm{mg}$ po once) and calamine lotion (topical use, tid). Mild hypokalemia was noted and was treated with oral potassium supplementation. Oral administration of a polysaccharideiron complex capsule $(0.15 \mathrm{~g})$ and a new motherwort capsule $(0.4 \mathrm{~g})$ for conduct iron supplementation and promote lochia discharge. The urinary catheter was removed on the day after surgery. The physician instructed the patient to observe whether her urination was normal. The dressing of the abdominal incision was changed. Symptom treatments, including incision care, fluid infusion, and enhancement of uterine contractility, were ordered. The patient's condition was closely monitored.

On the second day after surgery, the patient still complained of pruritus. She had no chills, fever, dizziness, or headaches. She was alert and had occasional pain due to contractions. After the removal of the catheter the previous day, the patient could urinate by herself, and she had no anal gas evacuation or bowel movement. Physical examination revealed that her vital signs were stable. Rashes could be seen on the patient's abdomen, back, and thighs. Heart and lung examinations were unremarkable. She had no breast engorgement. The abdominal incision appears clean and dry, without swelling or exudation. Her uterine fundus was at the level of the umbilicus. She had good uterine contractility and an intermediate amount of bloody lochia. After the ward round, the physician considered the patient's recovery to be on track and ordered the discontinuation of fluid infusion and the start of oral medications to promote uterine contractility. The patient was instructed to ambulate to promote the recovery of gastrointestinal function. Other care continued.
On the third day after surgery, the symptoms of pruritus were reduced. The patient had no chills, fever, dizziness, blurred vision, nausea, or vomiting. She was alert and had a good appetite. She occasionally experienced pain from uterine contractions and had no problems with urination. She had anal gas evacuation but no bowel movement. Physical examination revealed that her vital signs were stable. The rashes were improved compared to the previous day. Heart and lung examinations were unremarkable. The patient had slight engorgement in both breasts. Her abdomen was flat, and the abdominal incision was healing well, without redness, swelling, or exudation. Her uterine fundus was a finger-width below the umbilicus. She had good uterine contractility and a small amount of bloody lochia. After the ward round, the physician considered the patient's general condition to be acceptable and the healing of the surgical wound to be good. Oral drugs for the enhancement of uterine contractility and other care (particularly wound care) continued.

On the fourth day after surgery, the patient complained of mild pruritus on her right thigh. She has no chills, fever, cough, sputum, dizziness, or fatigue. She occasionally experienced pain from uterine contractions. Her general condition was acceptable. Her bowel movement and urination were normal. Physical examination showed that her vital signs were stable. The rashes had almost disappeared (Figure 2). Heart and lung examinations were unremarkable. She had slight engorgement of both breasts and no problem with breastfeeding. Her abdomen was flat, and the abdominal incision was healing well without redness, swelling, or exudation. Her uterine fundus was 2 finger-widths below the umbilicus. She had good uterine contractility and a small amount of bloody lochia. After the 
ward round, the physician considered the patient's general condition acceptable and the healing of the surgical wound satisfactory and ordered discharge.

The content involved here was following the ethical standards of the institutional and/or national research committee(s) and with the Helsinki Declaration (as revised in 2013). Written informed consent was obtained from the patient for publication of this manuscript and any accompanying images.

\section{Discussion}

Adverse skin reactions to drugs are common, affecting $2 \%$ to $3 \%$ of hospitalized patients. The drug-induced rash is the most common adverse skin reaction, accounting for $90 \%$ of all drug-related eruptions (1). The patient, in the present case, developed rashes after surgery considered the result of a drug allergy. Before the onset of the rashes, the medications used included cefuroxime sodium, morphine hydrochloride injection, ropivacaine hydrochloride injection, carboprost tromethamine. Adverse reactions of rashes have not been reported for ropivacaine hydrochloride injection and carboprost tromethamine in the relevant literature, and the association between these two drugs and rashes can be ruled out. Postoperative static drops of cefuroxime sodium, oxytocin injection, coenzyme Q10 sodium chloride, vitamin $\mathrm{C}$, compound amino acid injection (18aa-VII), new motherwort oral polysaccharide iron compound capsule, capsule, anti-inflammatory, hemostatic, inducing contractions, nutrition support therapy, these drugs are used only after the patient has itchy skin, so the rash caused by these drugs is not considered. According to the correlation between patient signs and drug injection time, the possibility of drug rash caused by cefuroxime sodium or morphine hydrochloride was greater.

Cefuroxime sodium is a member of the cephalosporin family of antibiotics, which are currently the most used antibacterial drugs in clinical practice. Studies $(2,3)$ have shown that the most common adverse reactions after using cephalosporin antibiotics are fever, pruritus, urticaria, and rash. The manufacturer's instructions also list rash as an adverse reaction. The mechanism underlying cephalosporin allergy is complex. The common precursor of these drugs is 7-aminocephalosporanic acid, which has the 7-position of the R1 side chain and 3-position of the R2 side chain. The final products of both alkaline hydrolysis and aminolysis are a derivative containing the $\mathrm{R} 1$ side chain. Therefore, the structure of the R1 side chain may play an essential role in the allergic reaction to cefuroxime sodium, while the R2 side chain and the 7-aminocephalosporan acid precursor are not related to allergy (4). Studies by Thong (5) and Pipet et al. (6) also showed that the R1 side chain could cause immunoglobulin IgE-mediated allergic reactions, which is the leading cause of allergic reactions to cephalosporin antibiotics. Proteins, impurities, and cephalosporin degradation products involved in drug production are also antigenic and can cause allergic reactions. The metabolites function as antigens or haptens to stimulate the body's immune system to produce corresponding antibodies, which cause allergic reactions when the drug re-enters the body.

Morphine is an opioid drug used for analgesic treatment. Morphine hydrochloride is a strong analgesic (7). Analgesia with morphine hydrochloride via the epidural space is a conventional treatment after the cesarean section. The optimal dose of morphine hydrochloride for epidural analgesia is one that can provide postoperative analgesia after cesarean section with minimal adverse reactions. A single bolus of morphine hydrochloride via the epidural approach supplies better analgesia than via the parenteral approach, but the effect is limited to the first day after cesarean section (8). Among the neurological adverse reactions to morphine, pruritus is one of the most troublesome problems. Mild pruritus can sometimes be ignored, but severe pruritus can affect daily life and recovery (9). Pregnant women are a special population and are more likely than other populations to experience pruritus caused by opioids. This may be due to the interaction of estrogen and opioid receptors. Singh et al. reported the incidence of pruritus caused by the epidural administration of $1.5 \mathrm{mg}$, morphine was $3 \%$, and the incidence of pruritus when $3 \mathrm{mg}$ morphine was administered was 50\% (10), while Mo et al. reported the incidence of intrathecal morphine-induced pruritus was $27.5 \%$ (11). Pruritus may occur $5.6 \pm 4.8$ hours after the administration of morphine and can last for $14.0 \pm 8.8$ hours. Also, Pettini et al. (12) and Tan et al. (13) reported a history of allergies is a potential risk factor for pruritus caused by morphine. Studies by Pettini et al. (12) and Aly et al. (14) have shown that serum serotonin levels are significantly increased in patients after cesarean section, and the absence of serotonin receptor antagonists is also a potential risk factor for pruritus.

Both allergens and opioids can cause pruritus in patients, but it is unclear whether there are common signaling pathways. Although the possible mechanism is unclear, experiments have shown that the occurrence of pruritus is related to the polymorphism of specific molecules. Tsai 
et al. (15) found that the $\mu$-opioid receptor gene (OPRM1) polymorphism is related to the occurrence of morphineinduced pruritus. It has been reported that the activation of opioid receptors leads to the release of histamine and causes skin reactions, including flushing, rash, urticaria, and pruritus (16). There is no prospective study on the treatment of opioid-induced pruritus. Although the role of histamines in opioid-induced pruritus is controversial, antihistamines are usually the first-line treatment for pruritus and can relieve pruritus to different extents. Treatment methods include the administration of antihistamines and using lowdose opioid antagonists, including naloxone $(4 \mu \mathrm{g}$, single dose). The dose can be repeated until the desired effect is achieved or until the pain worsens (17).

The patient was healthy before admission and had no history of drug or food allergies, and no other particular constitution susceptible to allergies. She did not use any drugs or allergenic foods before admission. Cephalosporins and opioids all may cause rashes and pruritus, and other adverse reactions. There are no reports that the combination of the two increases the incidence of rashes and pruritus. She developed rashes after using cefuroxime sodium and morphine hydrochloride. Each of these drugs can cause rashes. Symptoms can be alleviated after the discontinuation of medications and the treatment of symptoms. After patient stopped cefuroxime sodium, no other antibiotics were used. According to the evaluation standards of the association for monitoring adverse drug reactions in China, the time of use of these drugs is associated with the timing of the allergic reactions. Discontinuation of medications and the treatment of symptoms can improve the allergic reaction. Both cefuroxime sodium and morphine hydrochloride have been reported to cause allergic reactions. Excluding the influence of other factors, including diseases present before drug administration, suggests that the allergic reaction, here, may have been caused by cefuroxime sodium or morphine hydrochloride.

Early use of corticosteroids and antihistamines to prevent drug eruptions is generally not recommended in sensitized patients because there are currently no randomized trials evaluating the efficacy of prophylactic use of corticosteroids and antihistamines in preventing delayed drug response in sensitized patients. In terms of managing drug-related eruptions, identifying and stopping allergenic drugs is the first step of treatment. Patients with mild symptoms can gradually achieve symptom relief after stopping allergenic drugs without adding drug treatment. If the symptoms do not alleviate after discontinuation of medications, antihistamines or glucocorticoids can be added to relieve symptoms during the acute phase. When the lesions are widespread or in bullae form, the systemic use of antihistamines, glucocorticoids, and anti-infectives is needed, and topical application of ointments can hasten relief from the symptoms. Also, as the blood-brain barrier of infants has not been fully developed and has strong permeability, drugs are easy to pass through the blood-brain barrier and easily cause nervous system adverse reactions. Therefore, lactating women should avoid using drugs harmful to the nervous system. The first generation of antihistamines, including chlorpheniramine and ketotifen, have strong central nerve inhibition and should be avoided in lactating women. Loratadine is preferred among secondgeneration antihistamines due to its weaker CNS inhibition than azoastine and cetirizine. The American Academy of Pediatrics notes that maternal use of loratadine is usually not in conflict with breastfeeding. Therefore, loratadine is a reasonable choice for anti-allergy.

The patient had rashes on her body and extremities and experienced pruritus after using cefuroxime, sodium, and morphine. The physician identified the allergy-causing drugs promptly, discontinued them, and treated the patient with oral loratadine tablets and topical calamine lotion. Loratadine is a long-acting, potent, and highly selective histamine $\mathrm{H} 1$ receptor blocker. It has a rapid and longacting histamine inhibitory effect and can effectively relieve the pruritus symptoms of rash, and it also does not affect the patient's lactation. Calamine lotion contains calamine and zinc oxide and has anti-allergic and antipruritic effects. The combination of oral and topical anti-allergic and antipruritic drugs can promote the relief and subsidence of rashes.

Medical staff should fully grasp the indications and contraindications of cephalosporin antibiotics and morphine and choose drugs according to the patient's condition. If patients must use cephalosporin antibiotics and morphine, care should be taken to observe the patients' reactions following drug administration, and special attention should be paid to pregnant women and other special populations. Also, the patient's history of allergies should be decided in detail before using a drug, and changes in physical signs after drug administration should be closely monitored. If adverse reactions occur, the drug should be discontinued, and symptoms should be treated.

\section{Acknowledgments}

Funding: None. 


\section{Footnote}

Reporting Checklist: The authors have completed the CARE reporting checklist. Available at http://dx.doi.org/10.21037/ apm-20-1701

Conflicts of Interest: All authors have completed the ICMJE uniform disclosure form (available at http://dx.doi. org/10.21037/apm-20-1701). The authors have no conflicts of interest to declare.

Ethical Statement: The authors are accountable for all aspects of the work in ensuring that questions related to the accuracy or integrity of any part of the work are appropriately investigated and resolved. Written informed consent was obtained from the patient for publication of this manuscript and any accompanying images. The content involved here was following the ethical standards of the institutional and/or national research committee(s) and with the Helsinki Declaration (as revised in 2013).

Open Access Statement: This is an Open Access article distributed in accordance with the Creative Commons Attribution-NonCommercial-NoDerivs 4.0 International License (CC BY-NC-ND 4.0), which permits the noncommercial replication and distribution of the article with the strict proviso that no changes or edits are made and the original work is properly cited (including links to both the formal publication through the relevant DOI and the license). See: https://creativecommons.org/licenses/by-nc-nd/4.0/.

\section{References}

1. Bigby M. Rates of cutaneous reactions to drugs. Arch Dermatol 2001;137:765-70.

2. Magnolo N, Metze D, Ständer S. Pustulobullous variant of SDRIFE (symmetrical Drug related intertriginous and flexural exanthema). J Dtsch Dermatol Ges 2017;15:657-9.

3. Trautmann A, Benoit S, Goebeler M, et al. "Treating Through" Decision and Follow-up in Antibiotic TherapyAssociated Exanthemas. J Allergy Clin Immunol Pract 2017;5:1650-6.

4. Hasdenteufel F, Luyasu S, Renaudin JM, et al. Anaphylactic shock associated with cefuroxime axetil: structure-activity relationships. Ann Pharmacother 2007;41:1069-72.

5. Thong BY. Update on the management of antibiotic allergy. Allergy Asthma Immunol Res 2010;2:77-86.

6. Pipet A, Veyrac G, Wessel F, et al. A statement on cefazolin immediate hypersensitivity: data from a large database, and focus on the cross-reactivities. Clin Exp Allergy 2011;41:1602-8.

7. Macias MN, Hall TG, Ostlund J. Extended-release epidural morphine and postoperative nausea or vomiting. Am J Health Syst Pharm 2008;65:200.

8. Bonnet MP, Mignon A, Mazoit JX, et al. Analgesic Efficacy and Adverse Effects of Epidural Morphine Compared to Parenteral Opioids After Elective Caesarean Section: A Systematic Review. Eur J Pain 2010;14:894, e1-9.

9. Dominguez JE, Habib AS. Prophylaxis and treatment of the side-effects of neuraxial morphine analgesia following cesarean delivery. Curr Opin Anaesthesiol 2013;26:288-95.

10. Singh SI, Rehou S, Marmai KL, et al. The Efficacy of 2 Doses of Epi-dural Morphine for Postcesarean Delivery Analgesia: A Randomized Noninferiority Trial. Anesth Analg 2013;117:677-85.

11. Mo Y, Qiu S. Effects of dexmedetomidine in reducing post-cesarean adverse reactions. Exp Ther Med 2017;14:2036-9.

12. Pettini E, Micaglio M, Bitossi U, et al. Influence of OPRM1 polymorphism on postoperative pain after intrathecal morphine administration in Italian patients undergoing elective cesarean section. Clin J Pain 2018;34:178-81.

13. Tan X, Shen L, Wang L, et al. Incidence and Risk Factors for Epidural Morphine Induced Pruritus in Parturients Receiving Cesarean Section: A Prospective Multicenter Observational Study. Medicine (Baltimore) 2019;98:e17366.

14. Aly M, Ibrahim A, Farrag W, et al. Pruritus after intrathecal morphine for cesarean delivery: incidence, severity and its relation to serum serotonin level. Int J Obstet Anesth 2018;35:52-6.

15. Tsai FF, Fan SZ, Yang YM, et al. Human opioid (-receptor A118G polymorphism may protect against central pruritus by epidural morphine for post-cesarean analgesia. Acta Anaesthesiol Scand 2010;54:1265-9.

16. McNicol E, Horowicz-Mehler N, Fisk RA, et al. Management of opioid adverse reactions in cancer-related and chronic noncancer pain: a systematic review. J Pain 2003;4:231-56.

17. Ganesh A, Maxwell LG. Pathophysiology and management of opioid-induced pruritus. Drugs 2007;67:2323-33.

Cite this article as: Huang J, Zhu X, Jiang C, Liu Z, Zheng W. Rashes following cesarean delivery: a case report. Ann Palliat Med 2020;9(6):4353-4358. doi: 10.21037/apm-20-1701 